\title{
Year-round stratospheric aerosol backscatter ratios calculated from lidar measurements above northern Norway
}

\author{
Arvid Langenbach ${ }^{1}$, Gerd Baumgarten ${ }^{1}$, Jens Fiedler ${ }^{1}$, Franz-Josef Lübken ${ }^{1}$, Christian von Savigny ${ }^{2}$, and \\ Jacob Zalach ${ }^{2}$ \\ ${ }^{1}$ Leibniz-Institut für Atmosphärenphysik an der Universität Rostock, Schlossstraße 6, 18225 Kühlungsborn, Germany \\ ${ }^{2}$ Institut für Physik, Universität Greifswald, Felix-Hausdorff-Str. 6, 17489 Greifswald, Germany
}

Correspondence: Arvid Langenbach (langenbach@iap-kborn.de)

Received: 22 February 2019 - Discussion started: 7 March 2019

Revised: 13 June 2019 - Accepted: 1 July 2019 - Published: 24 July 2019

\begin{abstract}
We present a new method for calculating backscatter ratios of the stratospheric sulfate aerosol (SSA) layer from daytime and nighttime lidar measurements. Using this new method we show a first year-round dataset of stratospheric aerosol backscatter ratios at high latitudes. The SSA layer is located at altitudes between the tropopause and about $30 \mathrm{~km}$. It is of fundamental importance for the radiative balance of the atmosphere. We use a state-of-the-art Rayleigh-MieRaman lidar at the Arctic Lidar Observatory for Middle Atmosphere Research (ALOMAR) station located in northern Norway $\left(69^{\circ} \mathrm{N}, 16^{\circ} \mathrm{E} ; 380 \mathrm{~m}\right.$ a.s.l.). For nighttime measurements the aerosol backscatter ratios are derived using elastic and inelastic backscatter of the emitted laser wavelengths 355,532 and $1064 \mathrm{~nm}$. The setup of the lidar allows measurements with a resolution of about $5 \mathrm{~min}$ in time and $150 \mathrm{~m}$ in altitude to be performed in high quality, which enables the identification of multiple sub-layers in the stratospheric aerosol layer of less than $1 \mathrm{~km}$ vertical thickness.

We introduce a method to extend the dataset throughout the summer when measurements need to be performed under permanent daytime conditions. For that purpose we approximate the backscatter ratios from color ratios of elastic scattering and apply a correction function. We calculate the correction function using the average backscatter ratio profile at $355 \mathrm{~nm}$ from about $1700 \mathrm{~h}$ of nighttime measurements from the years 2000 to 2018 . Using the new method we finally present a year-round dataset based on about $4100 \mathrm{~h}$ of measurements during the years 2014 to 2017 .
\end{abstract}

\section{Introduction}

The importance of stratospheric sulfate aerosol (SSA) for the radiative balance and the ozone chemistry of the atmosphere is widely accepted. Long-term observations of the stratospheric aerosol layer are crucial for the analysis of global atmospheric temperature and ozone layer variability (Thomason and Peter, 2006; Solomon et al., 2011). The first in situ measurements of SSA were performed by Christian Junge and co-workers (Junge and Manson, 1961). They found a distinct layer between 15 and $25 \mathrm{~km}$ altitude with a peak at $20 \mathrm{~km}$ (Junge et al., 1961a, b). The stratospheric aerosol layer is therefore often referred to as the Junge layer. Remote sensing of the aerosol layer by lidar was started by Bartusek and Gambling (1971). Global satellite observations of SSA began in the late 1970s (e.g., Thomason and Peter, 2006; Kremser et al., 2016). The upper boundary of the SSA layer is determined by the evaporation of the aerosol particles due to rising temperatures in the stratosphere as well as sedimentation (Hofmann et al., 1985). The tropopause is the base of the aerosol layer since the upper tropospheric aerosol loads are often much lower than in the stratosphere (Kremser et al., 2016).

Understanding the formation and life cycle of SSA is impossible without understanding the processes controlling sulfur in the stratosphere. Stratospheric sulfur can be found in a broad variety of molecules, such as carbon disulfide $\left(\mathrm{CS}_{2}\right)$, sulfur dioxide $\left(\mathrm{SO}_{2}\right)$, carbonyl sulfide (OCS) and sulfuric acid $\left(\mathrm{H}_{2} \mathrm{SO}_{4}\right)$ (English et al., 2011). SSA typically consists of $75 \%$ sulfuric acid / water $\left(\mathrm{H}_{2} \mathrm{SO}_{4}-\mathrm{H}_{2} \mathrm{O}\right)$ solution droplets (Thomason and Peter, 2006). In volcanically quiescent periods, the main sources for these droplets are $\mathrm{CS}_{2}$ and OCS, 
which are emitted at the Earth's surface and lifted into the stratosphere by deep convection and the Brewer-Dobson circulation (Khaykin et al., 2017). They then react in multiple steps via $\mathrm{SO}_{2}$ into sulfuric acid (Kremser et al., 2016). Stratospheric aerosols are primarily washed out by sedimentation and through the quasi-isentropic transport of air masses in tropopause folds (Thomason and Peter, 2006).

Moreover, the SSA variability is dominated by major volcanic eruptions injecting sulfur directly into the stratosphere. These episodic, but powerful eruptions, can overlay the permanent stratospheric aerosol layer (referred to as "background" aerosol) for years and have a global cooling effect on the surface of the order of a few tenths of a degree Celsius (Robock and Mao, 1995). The fact that aerosols from large volcanic eruptions have global effects was first determined by worldwide observations of optical phenomena following the eruption of Krakatoa in 1883 (Symons, 1888). After the Mount Pinatubo eruption in 1991 the stratospheric sulfur burden was increased by a factor of 60 above background levels and remained elevated by a factor of 10 well into 1993 (McCormick et al., 1995).

The long-term development of SSA has been discussed in various studies (Kremser et al., 2016). Ignoring periods with volcanically enhanced SSA, observations covering the time span between 1970 and 2004 did not show significant changes in the background aerosol (Deshler et al., 2006). Newer studies show rising levels of SSA since 2002 (Hofmann et al., 2009; Vernier et al., 2011; Trickl et al., 2013; von Savigny et al., 2015). The reason for this increase is being debated. Originally the rise of the aerosol levels was connected to a fast increase in Asian sulfur emissions (Hofmann et al., 2009). More recent studies show an increase in non-volcanic aerosol inside of the Asian Tropopause Aerosol Layer (ATAL). This layer occurs during the northern summer above the Asian monsoon (Vernier et al., 2015; Yu et al., 2015). Vernier et al. (2011) showed, with the help of global satellite observations, that weaker eruptions also influence the stratospheric aerosol layer. These moderate eruptions are much less powerful compared to those of El Chichón or Mount Pinatubo, and the effect on stratospheric aerosol levels is much smaller. Nevertheless, several studies have shown that they have an impact on global surface temperatures (Solomon et al., 2011; Fyfe et al., 2013; Santer et al., 2014, 2015; Andersson et al., 2015).

Accurate long-term measurements are essential to quantify changes to the background, as well as volcanically and anthropogenically driven changes in the stratospheric aerosol layer. While there have been several reports on seasonaland decadal-scale ground-based lidar measurements of the aerosol layer at middle latitudes (Trickl et al., 2013; Khaykin et al., 2017; Zuev et al., 2017), there are no year-round or multi-year measurements of the stratospheric aerosol layer at high latitudes.

The main goal of this study is to present a year-round stratospheric aerosol record at polar latitudes for the first time, applying elastic laser scattering at three different wavelengths including measurements under full daylight conditions. This study introduces a method to approximate backscatter ratios of the stratospheric aerosol based on measurements of color ratios including a quantification of uncertainties. For this we use elastic and inelastic scattering measured during nighttime in the years 2000 to 2018 . To show the performance of the new method, we focus on a year-round dataset accumulated between 2014 and 2017. In Sect. 2 the instrumental setup and the data processing is described. Section 3 summarizes the extension of the dataset using measurements performed during permanent daylight in summer. In Sect. 4 we apply this new method to the years 2014 to 2017 and present a year-round climatology of SSA backscatter ratios.

\section{Instrument and method}

The Rayleigh-Mie-Raman lidar used in this study is installed at the Arctic Lidar Observatory for Middle Atmosphere Research (ALOMAR) in northern Norway $\left(69.3^{\circ} \mathrm{N}\right.$, $16.0^{\circ} \mathrm{E} ; 380 \mathrm{~m}$ a.s.1.). The lidar is employed for investigating the Arctic middle atmosphere in the 15 to $90 \mathrm{~km}$ altitude range (von Zahn et al., 2000). The instrument is optimized to measure atmospheric temperatures, winds and aerosols (Fiedler et al., 2008; Baumgarten, 2010). The lidar uses two power lasers and two receiving telescopes. Each of the two $\mathrm{Nd}$ :YAG power lasers generates 30 pulses per second with a pulse energy of 165,500 and $465 \mathrm{~mJ}$ at the wavelengths of 1064, 532 and $355 \mathrm{~nm}$, respectively. The telescopes have a diameter of $1.8 \mathrm{~m}$ and are tiltable from zenith pointing to $30^{\circ}$ off zenith in order to perform wind measurements and observations in a common volume with sounding rockets (Baumgarten et al., 2002).

The light collected by the telescopes is coupled alternatingly into the detection system synchronized to the alternatingly firing lasers. Figure 1 shows a schematic overview of the detection system, limited to the channels used in this study. The light is separated according to wavelength using dichroic beam splitters. The detection system is capable of detecting backscattered light at seven wavelengths during nighttime (elastic: 355, 532 and $1064 \mathrm{~nm}$; inelastic: 387, $529,530$ and $608 \mathrm{~nm})$. During daytime the system detects backscattered light at three elastic wavelengths $(355,532$ and $1064 \mathrm{~nm}$ ) using additional Fabry-Pérot etalon-based filters to reduce the solar background (von Zahn et al., 2000). The lidar measurements have been used before for calculating particle properties in the mesosphere and the upper stratosphere (e.g., von Cossart et al., 1999; Baumgarten et al., 2008; Gerding et al., 2003). For the study of aerosols in the stratosphere, the channels were extended with intensity cascaded detectors to allow for simultaneous measurements from the troposphere to the mesosphere. The backscatter signal is recorded 


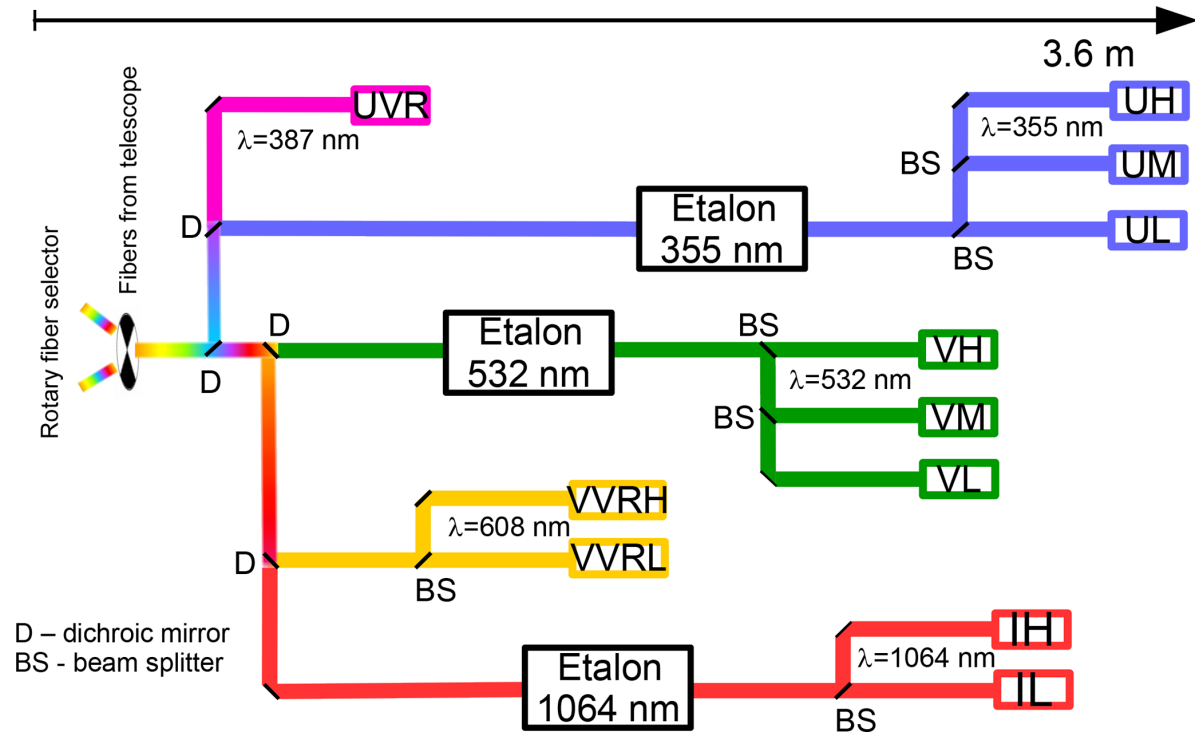

Figure 1. A simplified overview of the detection system of the lidar. The light collected by the two telescopes enters the detection system through a fiber selector (left) that is synchronized to the lasers. It is then separated according to wavelength with dichroic mirrors (D) and according to intensity by beam splitters (BS). In daylight conditions, the light is guided through etalons to suppress solar background. At the end of each detection branch, the photons are converted to electrical pulses using avalanche photodiodes and photomultiplier tubes. The names of these detectors are formed as follows: first letter represents the spectral range (U - ultraviolet, V - visible, I - infrared), and the last letter represents the sensitivity ( $\mathrm{L}$ - low, $\mathrm{M}$ - middle, $\mathrm{H}$ - high). Middle letters "VR" indicate vibrational Raman scattered light.

with a time and range resolution of $30 \mathrm{~s}$ and $50 \mathrm{~m}$, respectively.

\subsection{Processing of the raw data}

Before we start the actual aerosol retrieval we perform the following steps:

- Dead time correction. Once a photon is detected, minimum time span has to pass before another photon can be detected. This dead time $\tau$ is about 20 to $50 \mathrm{~ns}$ for the detectors used in this study. The corrected number of counted photons $N$ is calculated from the dead time and the count rate $N_{\mathrm{c}}$ (Kovalev and Eichinger, 2004):

$N=\frac{N_{\mathrm{c}}}{1-\tau \cdot N_{\mathrm{c}}}$.

- Background subtraction. The telescopes also collect light from scattered solar photons, stars or airglow. The mean signal from above $100 \mathrm{~km}$ represents this background signal since backscattered laser light from these altitudes is negligible. This mean is subtracted from the signals at lower altitudes.

- Gridding of lidar data. The raw data are averaged in time for $5 \mathrm{~min}$ and in altitude to bins of $150 \mathrm{~m}$ taking into account the different pointing angles of the telescopes. All altitudes in this work are referenced to the mean sea level.
- Correction for extinction by Rayleigh scattering. The intensity of the outgoing laser beam decreases slightly with altitude since a small fraction of the laser light is scattered by air molecules and aerosols. This also reduces the scattered, downward propagating light. The magnitude of this effect depends on the wavelength and the density of the atmosphere (Penndorf, 1957). We use air densities from a numerical weather prediction model (see below).

- Correction for extinction by ozone. A part of the laser light is absorbed by $\mathrm{O}_{3}$, in particular in the Chappuis bands affecting the laser wavelengths 532 and $608 \mathrm{~nm}$. This effect is corrected using the $\mathrm{O}_{3}$ absorption cross sections from Gorshelev et al. (2014) and the $\mathrm{O}_{3}$ mixing ratios and air density from a climatological model (see below).

- Combination of intensity cascaded detectors. We combine the signals of intensity cascaded detector groups by normalizing the lower intensity signal to the higher intensity signal in an altitude range in which both detectors provide a sufficient signal $S$ with a relative uncertainty of $\Delta S / S<0.1$.

The measurement uncertainty $\Delta S$ is calculated from the initial count of photons assuming a Poisson distribution. This uncertainty of the raw counts is then propagated through the processing steps listed above. 
We calculate the Rayleigh and ozone extinctions using densities and ozone mixing ratios provided by the European Centre for Medium-Range Weather Forecasts (ECMWF). The data from the Integrated Forecasting System of ECMWF are extracted for the location of ALOMAR on hourly basis. The model data are then interpolated to the lidar time and altitude bins $(5 \mathrm{~min}$ and $150 \mathrm{~m}$ ). We performed a sensitivity study using air densities from an empirical model (MSISE00; Picone et al., 2002) and a mean seasonal cycle for ozone (Rosenlof et al., 2015). It turns out that this leads to unrealistic backscatter ratios as the actual ozone profile significantly deviates from the climatological mean, especially in winter (see discussion of ozone extinction in Sect. 3).

The different detector groups and their corresponding scattering mechanisms are summarized in Table 1. An example for the signals of individual detectors and the combined signals $S^{\lambda}$ is shown in Fig. 2. The data are averaged for $17 \mathrm{~h}$ starting at 13:00 UT on 27 January 2018. About $7 \mathrm{~h}$ were performed under daytime conditions, while $10 \mathrm{~h}$ of the measurement were performed under nighttime conditions. The telescopes were pointing for about $1.5 \mathrm{~h}$ to zenith, and for the rest of the measurement were pointing at $20^{\circ}$ off zenith towards the north and east. In order to plot the data, we calculated the mean signals of the two telescopes. For the elastic scattered signals we observe a sudden increase in the signals below $30 \mathrm{~km}$, which is caused by tropospheric (below about $10 \mathrm{~km})$ and polar stratospheric clouds ( $\sim 15$ to $25 \mathrm{~km})$.

\subsection{Calculation of backscatter ratios}

The standard method to characterize the aerosol content in the atmosphere from lidar signals is to calculate the backscatter ratio $R$ from the molecule and aerosol backscatter coefficients $\beta_{\mathrm{m}}$ and $\beta_{\mathrm{a}}$, respectively (Fernald, 1984; Klett, 1985; Ansmann et al., 1990):

$R=\frac{\beta_{\mathrm{m}}+\beta_{\mathrm{a}}}{\beta_{\mathrm{m}}}$.

In our case the backscatter coefficients are proportional to the corrected signals $S$, shown in Fig. 2c. Let us for example consider scattering at $\lambda=1064 \mathrm{~nm}$ :

$R^{1064}=\frac{S_{\mathrm{m}}^{1064}+S_{\mathrm{a}}^{1064}}{S_{\mathrm{m}}^{1064}}=\frac{S^{1064}}{S_{\mathrm{m}}^{1064}}$.

The challenge is to retrieve the signal scattered by molecules only $\left(S_{\mathrm{m}}^{1064}\right)$ since the signal received by the lidar $\left(S^{1064}\right)$ contains both contributions from scattering on molecules and aerosols. For this we use the signal from Raman backscattering on $\mathrm{N}_{2}$ at $\lambda=387 \mathrm{~nm}$, which contains molecular scattering only: $\left(S^{387}\right)$. Since Raman scattering is less efficient compared to Rayleigh and Mie scattering, $S^{387}$ is much smaller compared to $S_{\mathrm{m}}^{1064}$ at any given altitude. However, when using the correction and applying the processing steps in Sect. 2.1, both signals are proportional to each other $\left(S^{387} \propto\right.$
$S_{\mathrm{m}}^{1064}$ ) since they are both given by molecular scattering. We determine the proportionality constant $F$ at an altitude $z_{F}$ in which no aerosols exist. Hence $S^{1064}$ equals $S_{\mathrm{m}}^{1064}$, which is typically the case above $34 \mathrm{~km}$ :

$F_{387}^{1064}=\left\langle\frac{S^{1064}}{S^{387}}\right\rangle_{z=z_{F}}$.

This allows the derivation of $S_{\mathrm{m}}^{1064}$ at any given altitude:

$S_{\mathrm{m}}^{1064}=F_{387}^{1064} \cdot S^{387}$.

Therefore Eq. (3) leads to

$R_{387}^{1064}=\frac{S^{1064}}{F_{387}^{1064} \cdot S^{387}}$.

This ratio is named $R_{387}^{1064}$ to indicate the wavelength of the elastic backscattered signal $(\lambda=1064 \mathrm{~nm})$ and the Raman wavelength $(\lambda=387 \mathrm{~nm})$. We use the altitude range $z_{F}$ from 34 to $38 \mathrm{~km}$ to determine $F_{387}^{1064}$ since an initial processing of the dataset showed that the aerosol layer sometimes reaches up to about $34 \mathrm{~km}$. In addition to this rather high normalization altitude we use a two-step process to reduce the effect of an aerosol layer reaching partly up into the normalization altitude range. First, we calculate the mean signal ratio in the normalization range, then we limit the data in the normalization range to those in which the signal ratio is within one standard deviation of the mean. More details on this are discussed in Sect. 4.

Additionally to $\lambda=1064 \mathrm{~nm}$, we also investigated the other two emitted wavelengths, namely $\lambda=532 \mathrm{~nm}$ and $\lambda=355 \mathrm{~nm}$. In total we derive three backscatter ratios: $R_{387}^{1064}, R_{387}^{532}$ and $R_{387}^{355}$, all as a function of altitude. Instead of the Raman signal at $\lambda=387 \mathrm{~nm}$ we have tentatively used another Raman signal, namely at a wavelength of $\lambda=608 \mathrm{~nm}$. It turned out, however, that this is not practical since this signal is partly absorbed by ozone (see Sect. 3). In this work, we focus on $R_{387}^{1064}$, i.e., $\lambda=1064 \mathrm{~nm}$ as the Rayleigh and Mie scattered signal, and $\lambda=387 \mathrm{~nm}$ as the Raman signal. This combination is superior to the others as ozone extinction does not affect these two wavelengths, and the backscatter ratio is found to be largest at $\lambda=1064 \mathrm{~nm}$.

\subsection{Identification of the stratospheric aerosol layer}

Only backscatter ratios from altitudes above the tropopause are analyzed in order to limit the data to the stratospheric aerosol layer. We calculate the dynamical and the thermal tropopause from ECMWF model data and select the higher value of the two as the lower-altitude limit for the backscatter ratio profiles.

We also remove measurements that show the presence of polar stratospheric clouds (PSCs) (Peter, 1997). From December to February these clouds occur frequently at our location. The calculated backscatter ratio of PSCs is about 1 order 
Table 1. Labels of combined signals and individual detectors. The name indicates in the first letter the spectral range (U - ultraviolet, V visible, I - infrared), and the last letter indicates the sensitivity ( $\mathrm{L}$ - low, M - middle, H-high). The letter pair "VR" indicates vibrational Raman scattered light. Indices represent the particular wavelength.

\begin{tabular}{llll}
\hline Combined signal & Detector & Scattering process & Daylight capability \\
\hline$S^{355}$ & $\mathrm{UL}_{355}$ & elastic (Rayleigh and Mie) & yes \\
& $\mathrm{UM}_{355}$ & & \\
& $\mathrm{UH}_{355}$ & & \\
\hline$S^{532}$ & $\mathrm{VL}_{532}$ & elastic (Rayleigh and Mie) & yes \\
& $\mathrm{VM}_{532}$ & & \\
& $\mathrm{VH}_{532}$ & & \\
\hline$S^{1064}$ & $\mathrm{IL}_{1064}$ & elastic (Rayleigh and Mie) & yes \\
& $\mathrm{IH}_{1064}$ & & \\
\hline$S^{387}$ & $\mathrm{UVR}_{387}$ & inelastic (Raman) & no \\
$S^{608}$ & VVRL $_{608}$ & inelastic (Raman) & no \\
& VVRH $_{608}$ & & \\
\hline
\end{tabular}
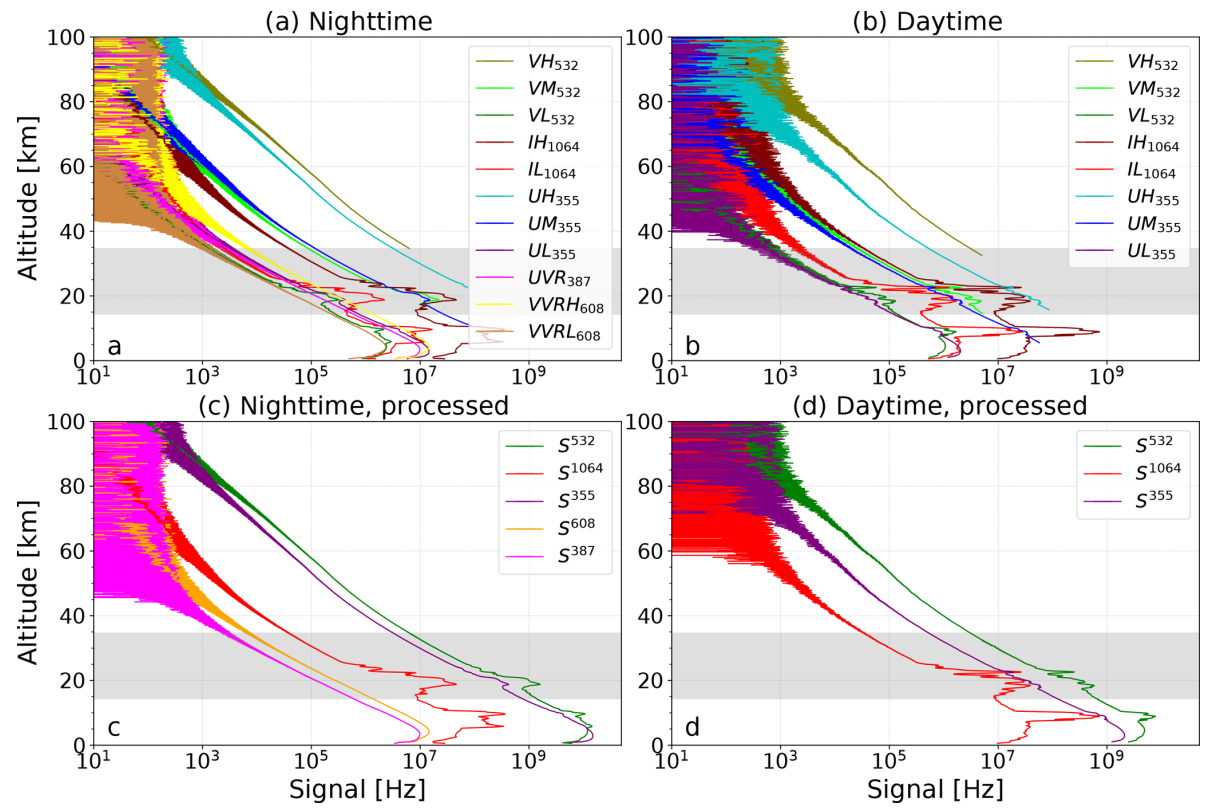

Figure 2. Time averaged altitude profiles of backscattered signals for a $17 \mathrm{~h}$ long measurement starting at 13:00 UT on 27 January 2018 . Panels (a) and (b) show the count rates of individual detectors. Panels (c) and (d) show the resulting profiles after combining the signals of the different detector groups. Panels (a) and (c) show about $10 \mathrm{~h}$ of nighttime measurements and panels (b) and (d) show about $7 \mathrm{~h}$ of daytime measurements. The approximate altitude range of the stratospheric aerosol layer is shown in gray.

of magnitude larger than that of the background aerosol (e.g., Fig. 2). Therefore we use a simple threshold of $R_{387}^{1064}>2.0$ to exclude PSCs from our dataset of stratospheric aerosols.

An example for a backscatter ratio $R_{387}^{1064}$ of the stratospheric aerosol layer from $88 \mathrm{~h}$ of measurements starting on 18 February 2018 is shown in Fig. 3. We observe a highly dynamic stratospheric aerosol layer consisting of multiple sublayers. There are several layers thinner than $1 \mathrm{~km}$ remaining separated and partially moving in parallel over several days. It should be emphasized that these layers are not connected to PSCs for two reasons: (1) the maximum backscatter ratio is well below the PSCs' threshold of $R_{387}^{1064}=2.0$, and (2) the temperatures at the altitudes of the layers were above $210 \mathrm{~K}$ and therefore about $15 \mathrm{~K}$ above formation temperature of PSCs (Beyerle and Neuber, 1994).

Figure 3 shows the evolution of the stratospheric aerosol layer during night and day. As the signal $S^{387}$ (needed to calculate $R_{387}^{1064}$ ) is not measured during daytime, we have calculated the mean signal $S^{387}$ during the night measurements (indicated in Fig. 3) and used this mean profile to cal- 


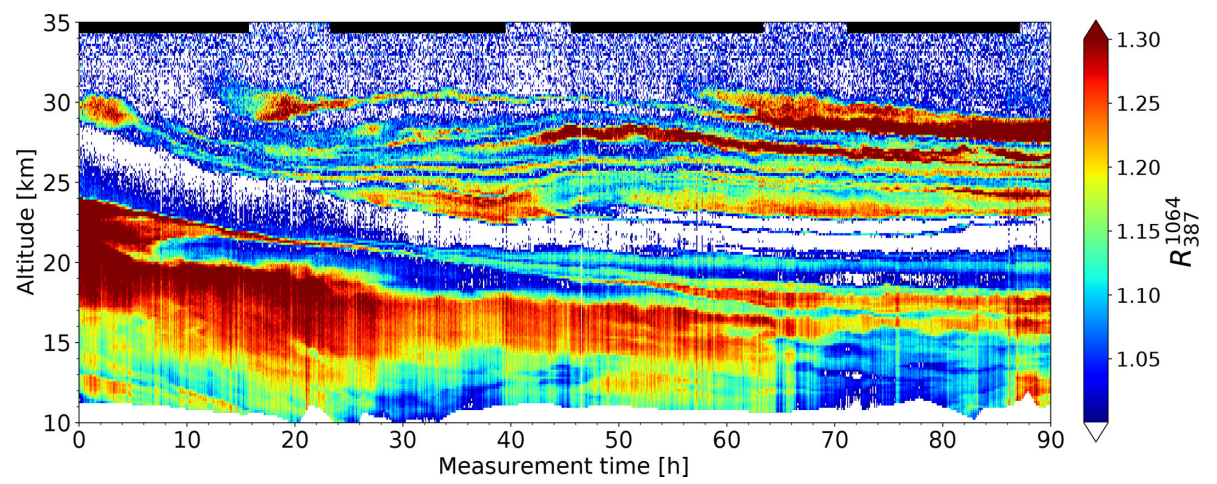

Figure 3. Stratospheric aerosol backscatter ratio $\left(R_{387}^{1064}\right)$ for a measurement starting at 15:30 UT on 18 February 2018 . The time resolution is $5 \mathrm{~min}$, and the altitude resolution is $150 \mathrm{~m}$. Black bars at the top indicate nighttime configuration. At the bottom end of about $11 \mathrm{~km}$ the data at altitudes below the tropopause are masked (white).

culate $R_{387}^{1064}$. Figure 3 shows that this method of calculating the backscatter ratio during daytime (using a nearby nighttime measurement) results in a reasonable evolution of the backscatter ratio.

\section{Calculating the backscatter ratio under daytime conditions}

The lidar is situated at $69.3^{\circ} \mathrm{N}$, i.e., north of the polar circle. At this latitude the daytime configuration of the lidar is used from about mid-May to mid-August. As shown in the previous section the backscatter ratio can be calculated for daytime measurements using a nearby nighttime observation. Unfortunately this is not possible during the summer months due to the permanent daylight. In order to retrieve a solid year-round dataset, we use the year-round available color ratio $C_{355}^{1064}$ with an empirical correction as a proxy for the backscatter ratio $R_{387}^{1064}$.

We define the "color ratio" $C$, namely the ratio of signals received from two of the three wavelengths $(1064,532$ and $355 \mathrm{~nm})$ normalized to the signal ratio at an altitude $\left(z_{F}\right)$ in which no aerosols exist. This is similar to the procedure for calculating the backscatter ratio in Sect. 2.2 and yields for 1064 and $355 \mathrm{~nm}$, as an example,

$C_{355}^{1064}=\frac{S^{1064}}{F_{355}^{1064} \cdot S^{355}}$.

It is worth noting that the definition of a color ratio used here is different from that used in von Cossart et al. (1999) and Baumgarten et al. (2008).

Figure 4 shows the mean backscatter ratio and color ratio profiles for a typical measurement in October 2015. The measurement lasted for $97 \mathrm{~h}$, thereof $46 \mathrm{~h}$ with daytime configuration and $51 \mathrm{~h}$ with nighttime configuration. Here, the backscatter ratio for the daytime part of the measurement is calculated by using the mean signal $S^{387}$ during nighttime observation as described in Sect. 2.3. Comparing $R_{387}^{1064}$

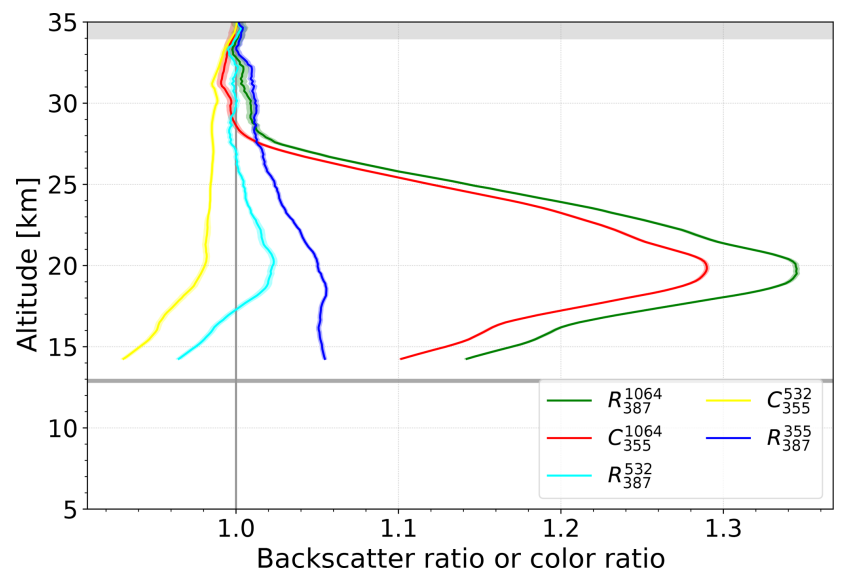

Figure 4. Backscatter ratio and color ratio profiles for a $97 \mathrm{~h}$ long measurement starting at 07:45 UT on 10 October 2015. About $46 \mathrm{~h}$ were measured with daytime configuration and $51 \mathrm{~h}$ with nighttime configuration. The gray area at the top indicates part of the normalization altitude. Shaded areas around the lines indicate the measurement uncertainty. The gray line at $13 \mathrm{~km}$ indicates the tropopause.

and $C_{355}^{1064}$, we see that $C_{355}^{1064}$ has nearly the same vertical structure but is about $5 \%$ lower than $R_{387}^{1064}$. The difference between $R_{387}^{1064}$ and $C_{355}^{1064}$ is primarily due to the aerosol backscatter signal $S_{\mathrm{a}}^{355}$ included in the signal $S^{355}$. In other words, the color ratio $C_{355}^{1064}$ is a proxy for $R_{387}^{1064}$ that deviates by less than about $5 \%$ from the true value of $R_{387}^{1064}$ at the peak of the stratospheric aerosol layer. We describe a method how to calculate the backscatter ratio at $1064 \mathrm{~nm}$ with respect to the molecular signal at $355 \mathrm{~nm}\left(R_{355}^{1064}\right)$ as follows:

$$
R_{355}^{1064}=\frac{S^{1064}}{F_{355}^{1064} S_{\mathrm{m}}^{355}} .
$$




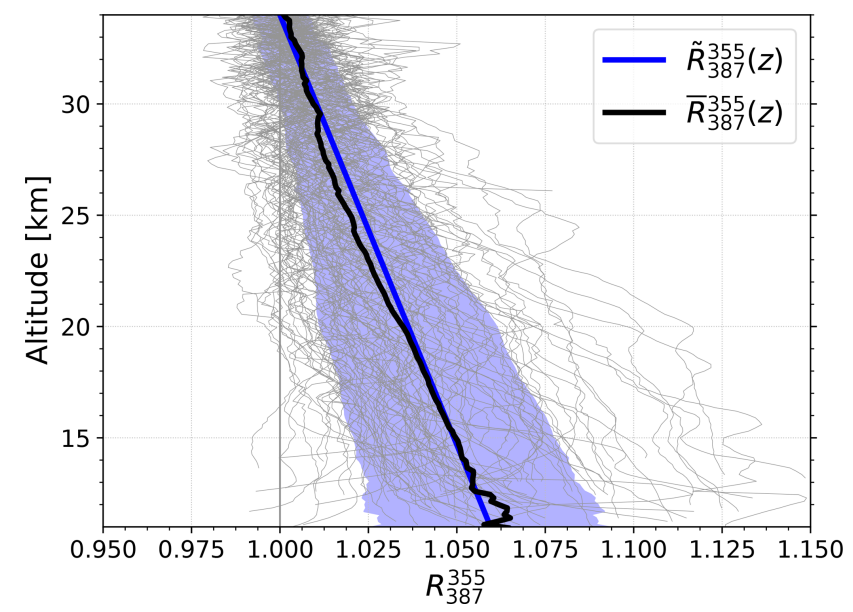

Figure 5. Backscatter ratios $R_{387}^{355}$ from nighttime measurements in the period 2000 to 2018. Each gray line represents the mean of the measurement run. The total measurement time is $1789 \mathrm{~h} . \bar{R}_{387}^{355}(z)$ is the mean of all profiles, and $\widetilde{R}_{387}^{355}(z)$ is a linear fit to $\bar{R}_{387}^{355}(z)$. The shaded area represents the standard deviation of the $R_{387}^{355}$ profiles.

This can be rewritten using the color ratio $C_{355}^{1064}$ and the backscatter ratio $R_{387}^{355}$,

$$
\begin{aligned}
R_{355}^{1064} & =\frac{S^{1064}}{F_{355}^{1064} F_{387}^{355} S^{387}} \\
& =\frac{S^{1064}}{F_{355}^{1064} S^{355}} \cdot \frac{S^{355}}{F_{387}^{355} S^{387}} \\
& =C_{355}^{1064} \cdot R_{387}^{355}
\end{aligned}
$$

Since the backscatter ratio $R_{387}^{355}$ is not available for daytime measurements, we approximate the actual profile by a mean of all available measurements to calculate the approximated backscatter ratio $R_{355}^{1064}$. Figure 5 shows the mean $R_{387}^{355}$ profile for each of the 103 measurements between 2000 and 2018 which cover a total of $1789 \mathrm{~h}$. These measurements were selected as they have a relative backscatter ratio uncertainty of less than one percent $\left(\Delta R_{387}^{355} / R_{387}^{355}<0.01\right)$.

The profiles of $R_{387}^{355}$ show a linear decrease with altitude towards $R=1$ at $z=34 \mathrm{~km}$. This behavior is seen very well in the mean profile $\bar{R}_{387}^{355}(z)$. We make use of this systematic altitude dependence by fitting a linear regression:

$\widetilde{R}_{387}^{355}(z)=\frac{z-407.95 \mathrm{~km}}{-374.16 \mathrm{~km}}$.

Finally, we use the fit $\widetilde{R}_{387}^{355}(z)$ to calculate the approximated backscatter ratio $R_{355}^{1064}$ :

$R_{355}^{1064}=C_{355}^{1064} \cdot \widetilde{R}_{387}^{355}(z)$.

Depending on altitude, the correction has a total effect on the backscatter ratio ranging from $5 \%$ at $15 \mathrm{~km}$ to zero

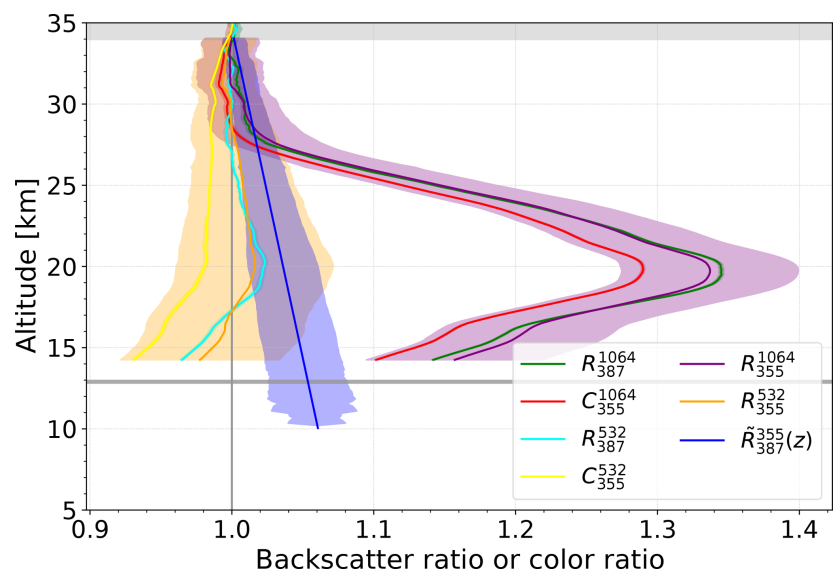

Figure 6. Backscatter ratio, color ratio and approximate backscatter ratio profiles $\left(R_{355}^{1064}, R_{355}^{532}\right)$ for a $97 \mathrm{~h}$ measurement starting 07:45 UT on 10 October 2015 (the same measurement as in Fig. 4). Colored shaded areas around the lines indicate the measurement uncertainty. Further explanations are given in Fig. 4.

at $34 \mathrm{~km}$. Figure 6 shows the approximate backscatter ratio $R_{355}^{1064}$ for the $97 \mathrm{~h}$ long measurement in October 2015. The approximated backscatter ratio profile $R_{355}^{1064}$ matches the backscatter ratio $R_{387}^{1064}$ by better than $1 \%$, which is well within the calculated uncertainty.

The uncertainty of the corrected profile is dominated by the uncertainty of the linear fit $\widetilde{R}_{387}^{355}(z)$. We have calculated the uncertainty of the fit as the standard deviation of the difference between $R_{387}^{1064}$ and $R_{355}^{1064}$ for each altitude. This difference is shown in Fig. 7 for each of the 103 measurements. It is symmetric over the whole altitude range and decreases with altitude. This behavior is as expected, as the effect of the correction tends to zero at $34 \mathrm{~km}$, and the $R_{387}^{355}$ profiles for each measurement tend to $R=1$ at $34 \mathrm{~km}$.

In the same way an approximated backscatter ratio $R_{355}^{532}$ is calculated from the corresponding color ratio $C_{355}^{532}$ and the fit $\widetilde{R}_{387}^{355}(z)$ :

$R_{355}^{532}=C_{355}^{532} \cdot \widetilde{R}_{387}^{355}(z)$.

Both $R_{387}^{1064}$ and $C_{355}^{1064}$ are not affected by ozone extinction whereas $R_{387}^{532}$ is affected by ozone extinction. We note that $R_{387}^{532}$ is smaller than 1 in limited altitude ranges $(z=15$ to $17 \mathrm{~km}$ ). By definition a backscatter ratio should not be smaller than 1 (Eq. 3). This indicates that the true ozone extinction may be different from that used for processing the data since the signal at $\lambda=532 \mathrm{~nm}$ is more strongly affected by ozone extinction than the signal at $\lambda=355 \mathrm{~nm}$. Due to the normalization of the backscatter ratio to 1 in the aerosolfree altitude $z_{F}$, an underestimation of ozone extinction reduces the backscatter ratio and may result in $R<1$. A similar effect arises due to a wavelength-dependent extinction of the aerosol layer. Here $R$ is reduced at lower altitudes if the wavelength of the elastic backscattered signal is more affected by aerosols than the Raman wavelength (see Eq. 6). 


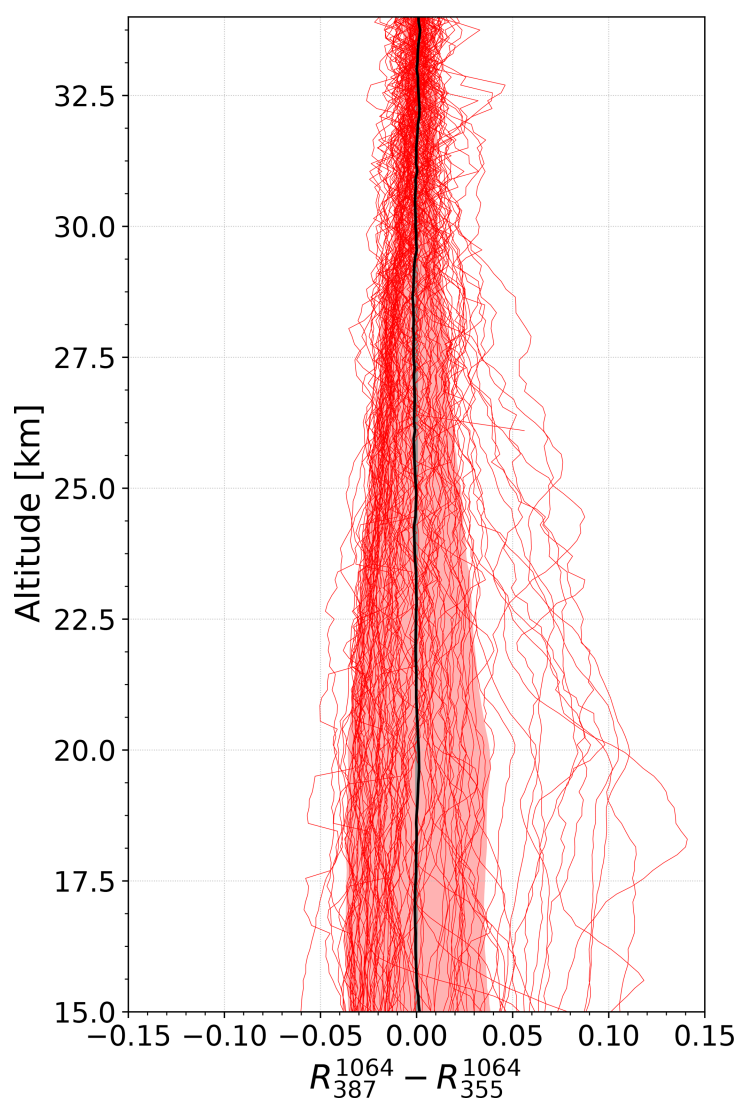

Figure 7. Difference in the backscatter ratio $R_{387}^{1064}$ and the approximated backscatter ratio $R_{355}^{1064}$ for 103 measurements between 2000 and 2018 where both ratios are available. The red shadowed area shows the standard deviation of the difference. The black line shows the mean difference.

The quality of the approximated backscatter ratio $R_{355}^{532}$ is also seen in Fig. 6 as it now agrees well with $R_{387}^{532}$. Notably $R_{355}^{532}$ is larger than 1 in most altitudes (in contrast to $C_{355}^{532}$ ). We would like to point out that approximated backscatter ratios underestimate the true backscatter ratios in cases of strong aerosol loads, and they overestimate the true backscatter ratios in cases of low aerosol loads since the correction function $\widetilde{R}_{387}^{355}(z)$ is derived from measurements with a mean aerosol load.

Although the backscatter ratios $R_{355}^{1064}$ are only marginally affected by the actual shape of the correction function $\widetilde{R}_{387}^{355}(z)$, it is worth discussing the linear decrease of $\widetilde{R}_{387}^{355}$ with altitude. First of all, we have not identified an instrumental problem that leads to this linear decrease with altitude; for example a incomplete overlap function would affect both $S^{355}$ and $S^{387}$ signals in the same way. Furthermore ozone extinction can be excluded as a potential error source since it has virtually no impact on these signals.

Using this approach a new dataset $R^{1064}$ is generated consisting of the exact backscatter ratio $R_{387}^{1064}$ for nighttime configuration (or daytime configuration with nearby night-

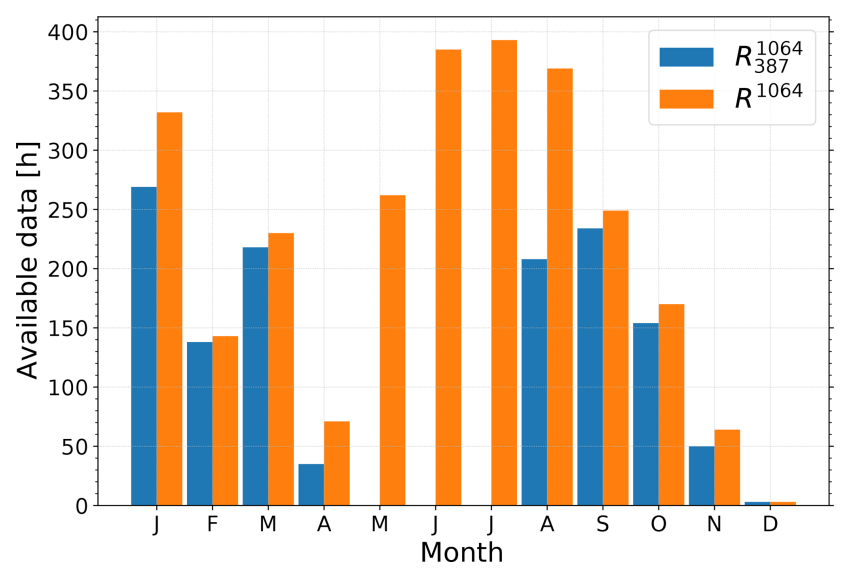

Figure 8. Available data in hours for each month for the years 2014 to 2017 for $R_{387}^{1064}$ (blue) and $R^{1064}$ (orange).

time measurements) and the approximated backscatter ratios $R_{355}^{1064}$ for the measurements in which only daytime configuration was applied (mostly summertime). The result is a complete year-round dataset of stratospheric aerosol backscatter ratio profiles.

\section{Results}

We applied the procedure described in Sect. 3 to the data from the years 2014 to 2017. During this period the lidar was operated for $4158 \mathrm{~h}$ and allowed the coverage of a typical seasonal cycle of the stratospheric aerosol. A total of 232 measurement runs were performed. In 24 of those runs PSCs were detected, and these runs were therefore excluded. The remaining 208 runs represent $3646 \mathrm{~h}$ of observations. Of these measurements $2391 \mathrm{~h}$ were performed with daytime configuration and $1255 \mathrm{~h}$ with nighttime configuration, respectively. Figure 8 shows the hours of measurements for the combined dataset $R^{1064}$ and the backscatter ratio $R_{387}^{1064}$. We see that the combined dataset includes the summer months in which no measurements of $R_{387}^{1064}$ are available. During the other months the $R^{1064}$ dataset is larger as well, compared to the $R_{387}^{1064}$ dataset.

We calculated the monthly mean backscatter ratios $R_{387}^{1064}$ and $R^{1064}$, omitting the month of December in which only $3 \mathrm{~h}$ of measurements are available. For this we first calculated hourly averaged backscatter ratios smoothed in altitude with a running mean of $1.1 \mathrm{~km}$. Then we calculated the average for the two telescopes. Finally the mean of the hourly profiles is calculated for each month. The standard error of the mean is given by $\sigma_{\mathrm{m}}=\sigma / \sqrt{n}$, where $\sigma$ is the standard deviation, and $n$ is the number of measurement hours per month.

The results for the nighttime backscatter ratio $R_{387}^{1064}$ from 79 measurement runs are shown in Fig. 9. We have excluded the month of April in the $R_{387}^{1064}$ dataset as the mean error between the tropopause and $34 \mathrm{~km}$ is $\overline{\sigma_{\mathrm{m}}}\left(R_{387}^{1064}\right)=0.025$. This 


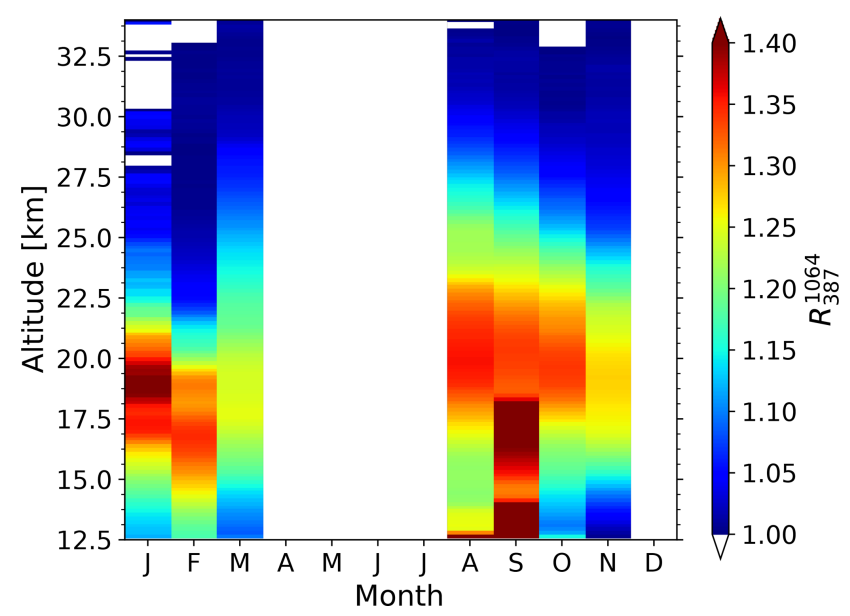

Figure 9. Monthly mean aerosol backscatter ratio $R_{387}^{1064}$ from 79 measurement runs from 2014 to 2017 . The dataset contains only nighttime measurements and includes a total of $1255 \mathrm{~h}$.

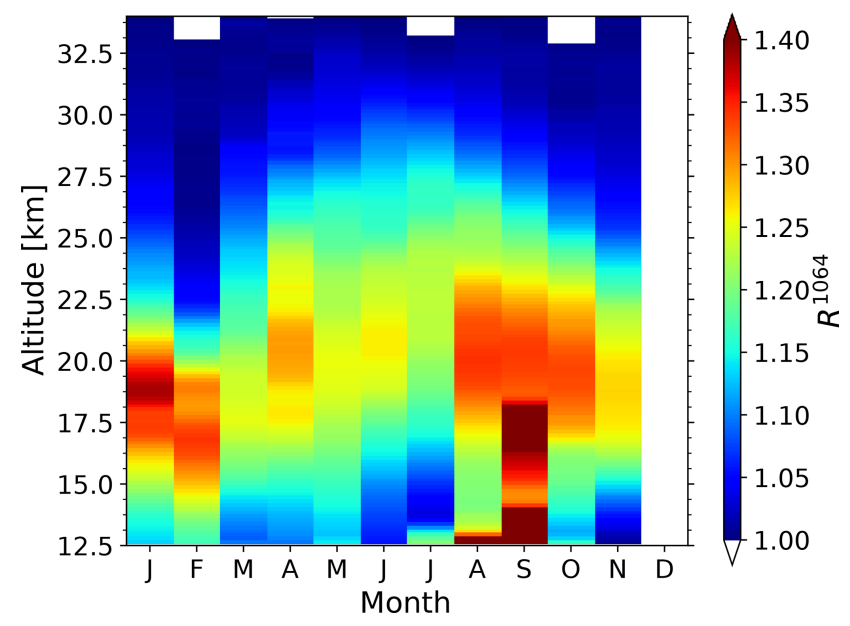

Figure 10. Monthly mean aerosol backscatter ratio $R^{1064}$ from 208 measurement runs from 2014 to 2017. The dataset contains night and daytime measurements, and it includes a total of $3646 \mathrm{~h}$.

value is rather high in comparison to the other months in which $\overline{\sigma_{\mathrm{m}}}\left(R_{387}^{1064}\right)$ is less than 0.01 .

The seasonal cycle for the combined dataset $R^{1064}$ from 208 measurement runs is shown in Fig. 10. Now the dataset also covers the summer months. In the months in which both datasets $R^{1064}$ and $R_{387}^{1064}$ are available, the datasets agree.

The uncertainties of the monthly mean backscatter ratios $R^{1064}$ result in $\sigma_{\mathrm{m}}\left(R^{1064}\right)=0.06$ at $11 \mathrm{~km}$ and $\sigma_{\mathrm{m}}\left(R^{1064}\right)=$ 0.02 at $34 \mathrm{~km}$ and are dominated by the uncertainty of the fit $\widetilde{R}_{387}^{355}(z)$.

The $R^{1064}$ and the $R_{387}^{1064}$ datasets both show enhanced aerosol backscatter in September in the lower stratosphere between 12 and $18 \mathrm{~km}$. These high aerosol levels originate from wildfires with strong pyrocumulonimbus activity over western Canada in September 2017. The smoke reached Eu- rope $10 \mathrm{~d}$ after its injection into the lower stratosphere. This event has been studied in detail by Ansmann et al. (2018).

During the winter months (November to March) the peak and the top of the aerosol layer is located at significantly lower altitudes compared to the rest of the year. In this period the peak of the layer is most often found below $22 \mathrm{~km}$. This is likely caused by the descending air within the polar vortex in winter. However, the location of the ALOMAR observatory is close to the edge of the polar vortex, so during the winter months we sometimes observe air within the vortex and sometimes outside the polar vortex. This results in a variation of the altitudes of the peak of the layer during the winter months.

A key finding of the new dataset $R^{1064}$ is that the stratospheric aerosol reaches well above $30 \mathrm{~km}$. In summer the typical backscatter ratio at $30 \mathrm{~km}$ is $R^{1064} \sim 1.05 \pm 0.02$. Therefore there is clear evidence for aerosols at this altitude. This finding is in contrast to previous studies in which the authors assumed an aerosol-free altitude starting at $30 \mathrm{~km}$ (McCormick et al., 1984; Barnes and Hofmann, 1997; Khaykin et al., 2017; Zuev et al., 2017). However, all of the previous studies were performed at mid to low latitudes.

\section{Summary and conclusions}

We have described the calculation of backscatter ratios using elastic (Rayleigh + Mie) and inelastic (Raman) scattering. For the investigation of the stratospheric aerosol, layer inelastic scattering is available only during nighttime. To our knowledge no lidar instrument exists that measures the stratospheric aerosol during daytime using the classical Raman method of calculating a backscatter ratio from elastic and inelastic scattering.

An extension of the backscatter ratio time series to daytime using close-in-time nighttime inelastic signals allows the observation of small-scale structures present in the stratospheric aerosol even during daytime. We present for the first time multiple sharp background aerosol layers of less than $1 \mathrm{~km}$ vertical thickness that partly move in parallel to each other over several days.

In order to calculate the backscatter ratios $R^{1064}$ and $R^{532}$ during daytime when Raman signals are not available, we have developed a proxy that is based on the measured color ratio of elastic scattering at the wavelengths 1064, 532 and $355 \mathrm{~nm}$, and an empirical correction function. The color ratios with respect to $\lambda=355 \mathrm{~nm}$ already yield reasonable values for the backscatter ratios. However, the color ratios are about $5 \%$ smaller than the backscatter ratios.

A correction function was calculated from multiple measurements of the backscatter ratio $R_{387}^{355}$ in the period from 2000 to 2018. The measurements show a linear decrease of $R_{387}^{355}$ with altitude. The largest uncertainties on the monthly mean approximated backscatter ratio $\left(R^{1064}\right.$ and $\left.R^{532}\right)$ are found in the lowest altitudes of the stratospheric aerosol 
layer, but even there we have observed that the approximated backscatter ratio deviates from the true backscatter ratio by less than $1 \%$.

Using this correction function we calculated for the first time a seasonal cycle of backscatter ratios at high latitudes including the summer months. A dedicated seasonal cycle of the peak of the aerosol layer with higher altitudes in summer and lower altitudes in winter is found. The top altitude of the layer varies in a similar way throughout the year. The aerosol reaches as high as $34 \mathrm{~km}$ during the summer months.

We propose to use this new method of calculating the backscatter ratio of the stratospheric aerosol layer from pure elastic scattering for the study of decadal-scale variations at high latitudes. Furthermore, the study of variations in stratospheric aerosol at the smallest scales detectable $(\mathrm{d} t<5$ minutes, $\mathrm{d} z<150 \mathrm{~m}$ ) will benefit from the method as elastic scattering provides a better signal-to-noise ratio during nighttime and daytime.

Code and data availability. The datasets used in this study can be obtained by contacting the first author.

Author contributions. AL and GB developed the retrieval technique for backscatter ratios at daytime and nighttime and drafted the paper. AL performed the data analysis. JF, FJL, JZ and CvS provided guidance in all elements of this work. All authors contributed toward revising and improving the paper.

Competing interests. The authors declare that they have no conflict of interest.

Acknowledgements. This work benefited from the excellent support of Jens Hildebrand, Michael Gerding and the dedicated staff at the ALOMAR observatory. The European Centre for Medium-Range Weather Forecasts (ECMWF) is gratefully acknowledged for providing the data.

Financial support. This research has been supported by the Deutsche Forschungsgesellschaft (grant no. SA-1351/7).

The publication of this article was funded by the Open Access Fund of the Leibniz Association.

Review statement. This paper was edited by Andrew Sayer and reviewed by Alain Hauchecorne and two anonymous referees.

\section{References}

Andersson, S. M., Martinsson, B. G., Vernier, J.-P., Friberg, J., Brenninkmeijer, C. A. M., Hermann, M., van Velthoven, P.
F. J., and Zahn, A.: Significant radiative impact of volcanic aerosol in the lowermost stratosphere, Nat. Commun., 6, 7692, https://doi.org/10.1038/ncomms8692, 2015.

Ansmann, A., Riebesell, M., and Weitkamp, C.: Measurement of atmospheric aerosol extinction profiles with a Raman lidar, Opt. Lett., 15, 746-748, https://doi.org/10.1364/ol.15.000746, 1990.

Ansmann, A., Baars, H., Chudnovsky, A., Mattis, I., Veselovskii, I., Haarig, M., Seifert, P., Engelmann, R., and Wandinger, U.: Extreme levels of Canadian wildfire smoke in the stratosphere over central Europe on 21-22 August 2017, Atmos. Chem. Phys., 18, 11831-11845, https://doi.org/10.5194/acp-18-118312018, 2018.

Barnes, J. E. and Hofmann, D. J.: Lidar measurements of stratospheric aerosol over Mauna Loa Observatory, Geophys. Res. Lett., 24, 1923-1926, https://doi.org/10.1029/97gl01943, 1997.

Bartusek, K. and Gambling, D.: Simultaneous measurements of stratospheric aerosols using lidar and the twilight technique, J. Atmos. Terr. Phys., 33, 1415-1430, https://doi.org/10.1016/0021-9169(71)90013-4, 1971.

Baumgarten, G.: Doppler Rayleigh/Mie/Raman lidar for wind and temperature measurements in the middle atmosphere up to $80 \mathrm{~km}$, Atmos. Meas. Tech., 3, 1509-1518, https://doi.org/10.5194/amt-3-1509-2010, 2010.

Baumgarten, G., Lübken, F.-J., and Fricke, K. H.: First observation of one noctilucent cloud by a twin lidar in two different directions, Ann. Geophys., 20, 1863-1868, https://doi.org/10.5194/angeo-20-1863-2002, 2002.

Baumgarten, G., Fiedler, J., Lübken, F.-J., and von Cossart, G.: Particle properties and water content of noctilucent clouds and their interannual variation, J. Geophys. Res., 113, D06203, https://doi.org/10.1029/2007jd008884, 2008.

Beyerle, G. and Neuber, R.: The stratospheric aerosol content above Spitzbergen during winter 1991/92, Geophys. Res. Lett., 21, 1291-1294, https://doi.org/10.1029/93gl03292, 1994.

Deshler, T., Anderson-Sprecher, R., Jäger, H., Barnes, J., Hofmann, D. J., Clemesha, B., Simonich, D., Osborn, M., Grainger, R. G., and Godin-Beekmann, S.: Trends in the nonvolcanic component of stratospheric aerosol over the period 1971-2004, J. Geophys. Res., 111, D01201, https://doi.org/10.1029/2005jd006089, 2006.

English, J. M., Toon, O. B., Mills, M. J., and Yu, F.: Microphysical simulations of new particle formation in the upper troposphere and lower stratosphere, Atmos. Chem. Phys., 11, 9303-9322, https://doi.org/10.5194/acp-11-9303-2011, 2011.

Fernald, F. G.: Analysis of atmospheric lidar observations: some comments, Appl. Opt., 23, 652-653, https://doi.org/10.1364/ao.23.000652, 1984.

Fiedler, J., Baumgarten, G., and von Cossart, G.: A middle atmosphere lidar for multi-parameter measurements at a remote site, 24th ILRC, 824-827, 2008.

Fyfe, J. C., von Salzen, K., Cole, J. N. S., Gillett, N. P., and Vernier, J.-P.: Surface response to stratospheric aerosol changes in a coupled atmosphere-ocean model, Geophys. Res. Lett., 40, 584-588, https://doi.org/10.1002/grl.50156, 2013.

Gerding, M., Baumgarten, G., Blum, U., Thayer, J. P., Fricke, K.H., Neuber, R., and Fiedler, J.: Observation of an unusual midstratospheric aerosol layer in the Arctic: possible sources and implications for polar vortex dynamics, Ann. Geophys., 21, 10571069, https://doi.org/10.5194/angeo-21-1057-2003, 2003. 
Gorshelev, V., Serdyuchenko, A., Weber, M., Chehade, W., and Burrows, J. P.: High spectral resolution ozone absorption crosssections - Part 1: Measurements, data analysis and comparison with previous measurements around $293 \mathrm{~K}$, Atmos. Meas. Tech., 7, 609-624, https://doi.org/10.5194/amt-7-609-2014, 2014.

Hofmann, D., Barnes, J., O’Neill, M., Trudeau, M., and Neely, R.: Increase in background stratospheric aerosol observed with lidar at Mauna Loa Observatory and Boulder, Colorado, Geophys. Res. Lett., 36, L15808, https://doi.org/10.1029/2009gl039008, 2009.

Hofmann, D. J., Rosen, J. M., and Gringel, W.: Delayed production of sulfuric acid condensation nuclei in the polar stratosphere from El Chichon volcanic vapors, J. Geophys. Res., 90, 2341, https://doi.org/10.1029/jd090id01p02341, 1985.

Junge, C. E. and Manson, J. E.: Stratospheric aerosol studies, J. Geophys. Res., 66, 2163-2182, https://doi.org/10.1029/jz066i007p02163, 1961.

Junge, C. E., Chagnon, C. W., and Manson, J. E.: Stratospheric Aerosols, J. Meteorol., 18, 81-108, https://doi.org/10.1175/1520-0469(1961)018<0081:sa>2.0.co;2, 1961a.

Junge, C. E., Chagnon, C. W., and Manson, J. E.: A Worldwide Stratospheric Aerosol Layer, Science, 133, 1478-1479, https://doi.org/10.1126/science.133.3463.1478-a, 1961b.

Khaykin, S. M., Godin-Beekmann, S., Keckhut, P., Hauchecorne, A., Jumelet, J., Vernier, J.-P., Bourassa, A., Degenstein, D. A., Rieger, L. A., Bingen, C., Vanhellemont, F., Robert, C., DeLand, M., and Bhartia, P. K.: Variability and evolution of the midlatitude stratospheric aerosol budget from 22 years of ground-based lidar and satellite observations, Atmos. Chem. Phys., 17, 18291845, https://doi.org/10.5194/acp-17-1829-2017, 2017.

Klett, J. D.: Lidar inversion with variable backscatter/extinction ratios, Appl. Opt., 24, 1638, https://doi.org/10.1364/ao.24.001638, 1985.

Kovalev, V. A. and Eichinger, W. E.: Elastic Lidar, John Wiley \& Sons, Inc., Hoboken, https://doi.org/10.1002/0471643173, 2004.

Kremser, S., Thomason, L. W., von Hobe, M., Hermann, M., Deshler, T., Timmreck, C., Toohey, M., Stenke, A., Schwarz, J. P., Weigel, R., Fueglistaler, S., Prata, F. J., Vernier, J.-P., Schlager, H., Barnes, J. E., Antuña-Marrero, J.-C., Fairlie, D., Palm, M., Mahieu, E., Notholt, J., Rex, M., Bingen, C., Vanhellemont, F., Bourassa, A., Plane, J. M. C., Klocke, D., Carn, S. A., Clarisse, L., Trickl, T., Neely, R., James, A. D., Rieger, L., Wilson, J. C., and Meland, B.: Stratospheric aerosol-Observations, processes, and impact on climate, Rev. Geophys., 54, 278-335, https://doi.org/10.1002/2015rg000511, 2016.

McCormick, M., Swissler, T., Fuller, W., Hunt, W., and Osborn, M.: Airborne and ground-based lidar measurements of the El Chichon stratospheric aerosol from $90 \mathrm{~N}$ to $56 \mathrm{~S}$, Geofis. Int., 23, 187-221, 1984.

McCormick, M. P., Thomason, L. W., and Trepte, C. R.: Atmospheric effects of the Mt Pinatubo eruption, Nature, 373, 399404, https://doi.org/10.1038/373399a0, 1995.

Penndorf, R.: Tables of the Refractive Index for Standard Air and the Rayleigh Scattering Coefficient for the Spectral Region between 02 and $200 \mu$ and Their Application to Atmospheric Optics, J. Opt. Soc. Am., 47, 176, https://doi.org/10.1364/josa.47.000176, 1957.
Peter, T.: Microphysics and heterogeneous chemistry of polar stratospheric clouds, Ann. Rev. Phys. Chem., 48, 785-822, https://doi.org/10.1146/annurev.physchem.48.1.785, 1997.

Picone, J. M., Hedin, A. E., Drob, D. P., and Aikin, A. C.: NRLMSISE-00 empirical model of the atmosphere: Statistical comparisons and scientific issues, J. Geophys. Res.-Space, 107, 1468, https://doi.org/10.1029/2002ja009430, 2002.

Robock, A. and Mao, J.: The Volcanic Signal in Surface Temperature Observations, J. Climate, 8, 1086-1103, https://doi.org/10.1175/15200442(1995)008<1086:tvsist>2.0.co;2, 1995.

Rosenlof, K., Hassler, B., Bodeker, G., and NOAA CDR Program: NOAA Climate Data Record (CDR) of Zonal Mean Ozone Binary Database of Profiles (BDBP), version 1.0, https://doi.org/10.7289/v56m34rt, 2015.

Santer, B. D., Bonfils, C., Painter, J. F., Zelinka, M. D., Mears, C., Solomon, S., Schmidt, G. A., Fyfe, J. C., Cole, J. N. S., Nazarenko, L., Taylor, K. E., and Wentz, F. J.: Volcanic contribution to decadal changes in tropospheric temperature, Nat. Geosci., 7, 185-189, https://doi.org/10.1038/ngeo2098, 2014.

Santer, B. D., Solomon, S., Bonfils, C., Zelinka, M. D., Painter, J. F., Beltran, F., Fyfe, J. C., Johannesson, G., Mears, C., Ridley, D. A., Vernier, J.-P., and Wentz, F. J.: Observed multivariable signals of late 20 th and early 21 st century volcanic activity, Geophys. Res. Lett., 42, 500-509, https://doi.org/10.1002/2014g1062366, 2015.

Solomon, S., Daniel, J. S., Neely, R. R., Vernier, J.-P., Dutton, E. G., and Thomason, L. W.: The Persistently Variable "Background" Stratospheric Aerosol Layer and Global Climate Change, Science, 333, 866-870, https://doi.org/10.1126/science.1206027, 2011.

Symons, G.: The eruption of Krakatoa, and subsequent phenomena: report of the Krakatoa committee of the Royal Society, printed by Harrison and Sons, London, https://doi.org/10.3931/e-rara16337,1888

Thomason, L. and Peter, T. (Eds.):SPARC Assessment of Stratospheric Aerosol Properties (ASAP), Tech. rep., SPARC Office, available at: http://www.sparc-climate.org/publications/ sparc-reports/ (last access: 15 January 2019), 2006.

Trickl, T., Giehl, H., Jäger, H., and Vogelmann, H.: 35 yr of stratospheric aerosol measurements at Garmisch-Partenkirchen: from Fuego to Eyjafjallajökull, and beyond, Atmos. Chem. Phys., 13, 5205-5225, https://doi.org/10.5194/acp-13-5205-2013, 2013.

Vernier, J.-P., Thomason, L. W., Pommereau, J.-P., Bourassa, A., Pelon, J., Garnier, A., Hauchecorne, A., Blanot, L., Trepte, C., Degenstein, D., and Vargas, F.: Major influence of tropical volcanic eruptions on the stratospheric aerosol layer during the last decade, Geophys. Res. Lett., 38, L12807, https://doi.org/10.1029/2011g1047563, 2011.

Vernier, J.-P., Fairlie, T. D., Natarajan, M., Wienhold, F. G., Bian, J., Martinsson, B. G., Crumeyrolle, S., Thomason, L. W., and Bedka, K. M.: Increase in upper tropospheric and lower stratospheric aerosol levels and its potential connection with Asian pollution, J. Geophys. Res.-Atmos., 120, 1608-1619, https://doi.org/10.1002/2014jd022372, 2015.

von Cossart, G., Fiedler, J., and von Zahn, U.: Size distributions of NLC particles as determined from 3-color observations of NLC by ground-based lidar, Geophys. Res. Lett., 26, 1513-1516, https://doi.org/10.1029/1999g1900226, 1999. 
von Savigny, C., Ernst, F., Rozanov, A., Hommel, R., Eichmann, K.U., Rozanov, V., Burrows, J. P., and Thomason, L. W.: Improved stratospheric aerosol extinction profiles from SCIAMACHY: validation and sample results, Atmos. Meas. Tech., 8, 5223-5235, https://doi.org/10.5194/amt-8-5223-2015, 2015.

von Zahn, U., von Cossart, G., Fiedler, J., Fricke, K. H., Nelke, G., Baumgarten, G., Rees, D., Hauchecorne, A., and Adolfsen, K.: The ALOMAR Rayleigh/Mie/Raman lidar: objectives, configuration, and performance, Ann. Geophys., 18, 815-833, https://doi.org/10.1007/s00585-000-0815-2, 2000.
Yu, P., Toon, O. B., Neely, R. R., Martinsson, B. G., and Brenninkmeijer, C. A. M.: Composition and physical properties of the Asian Tropopause Aerosol Layer and the North American Tropospheric Aerosol Layer, Geophys. Res. Lett., 42, 2540-2546, https://doi.org/10.1002/2015gl063181, 2015.

Zuev, V. V., Burlakov, V. D., Nevzorov, A. V., Pravdin, V. L., Savelieva, E. S., and Gerasimov, V. V.: 30-year lidar observations of the stratospheric aerosol layer state over Tomsk (Western Siberia, Russia), Atmos. Chem. Phys., 17, 3067-3081, https://doi.org/10.5194/acp-17-3067-2017, 2017. 